\title{
Association of British Neurologists: revised (2015) guidelines for prescribing disease-modifying treatments in multiple sclerosis
}

\author{
Neil Scolding, ${ }^{1}$ David Barnes, ${ }^{2}$ Sarah Cader, ${ }^{3}$ Jeremy Chataway, ${ }^{4}$ \\ Abhijit Chaudhuri, ${ }^{5}$ Alasdair Coles, ${ }^{6}$ Gavin Giovannoni, ${ }^{7}$ David Miller, ${ }^{8}$ \\ Waqar Rashid, ${ }^{9}$ Klaus Schmierer, ${ }^{10}$ Abdullah Shehu, ${ }^{11}$ Eli Silber, ${ }^{12}$ \\ Carolyn Young, ${ }^{13}$ John Zajicek $^{14}$
}

\begin{abstract}
- Additional material is published online only. To view please visit the journal online (http://dx.doi.org/10.1136/ practneurol-2015-001139)

For numbered affiliations see end of article.
\end{abstract}

\section{Correspondence to} Professor Neil Scolding, Bristol Institute of Clinical Neurosciences, Learning and Research Building, Southmead Hospital, Bristol BS10 5NB, UK; n.j.scolding@bristol.ac.uk

Accepted 27 May 2015 Published Online First 22 June 2015

\section{INTRODUCTION}

In June 1999, the Association of British Neurologists (ABN) first published guidelines for the use of the licensed multiple sclerosis (MS) disease-modifying treatments (at that time $\beta$-interferon and glatiramer acetate). The guidelines were revised in 2001 and have been periodically updated since then. In 2002, following the negative assessment of these treatments by the National Institute for Health and Care Excellence (NICE), the MS risk-sharing scheme started, in which patients eligible according to the 2001 $A B N$ guidelines were provided with treatment funded through the UK National Health Service (NHS), and monitored annually for up to 10 years. ${ }^{1}$ Recruitment to the risk-sharing scheme cohort is complete. Pending a future final evaluation, the UK Department of Health's instruction to NHS funders remains in place: that patients who fulfil the ABN criteria should continue to receive treatment funded through the NHS. The British neurological community has fully accepted the risk-sharing scheme for prescribing $\beta$-interferon and glatiramer acetate. Approximately 70 'treating centres' have recruited $>5000$ patients between 2002 and 2005, and these have been monitored annually for 10 years; many more patients have received these treatments since 2005. The ABN published revised guidelines in 2007, and then again in 2009, following the licensing of natalizumab and mitoxantrone.

This 2015 revised guideline replaces former versions. It includes all newly approved or licensed treatments for MS and represents a consensus concerning their use. These guidelines will require future revision as other treatments receive approval (eg, daclizumab and ocrelizumab): we suggest they are reviewed after an interval of no longer than 12 months. The guideline is not intended to provide a complete description of the possible complications and monitoring of diseasemodifying treatments in MS; we refer prescribing neurologists to the relevant summaries of product characteristics.

\section{BACKGROUND}

MS is a complex disease. Perhaps uniquely in neurology its clinical course is characterised by two quite different clinical phases: the relapsing-remitting phase and a phase of progressive disease. The relationship between relapse and progression is not well understood. The clinical picture is made more complicated by the fact that a minority of patients experience only one or the other of these 'phases'; while in the remaining majority of patients, the phases overlap, though over varying lengths of time. Given (1) this unpredictable period of overlap and (2) that relapses differ significantly in their duration and (3) that in progressive disease the rate of deterioration can vary substantially (even longitudinally in the same individual), it can be difficult to mark the transition from relapsing-remitting disease to progressive MS, so this is commonly done only in retrospect.

In the individual patient, MS remains a fundamentally unpredictable condition. There are many factors that influence prognosis and disease course, including 
sex, relapse frequency, type of relapse, age, MRI lesion load and spinal cord involvement. However, for most patients, MS results in a gradual accumulation of fixed neurological disability over time. There is an increasing tendency to use treatment earlier and more aggressively in those people deemed to have a worse outlook, but there is limited direct evidence of long-term benefit for making such decisions. The ABN strongly supports the collection of long-term and collaborative research data to facilitate better patient-doctor decisions.

Disease-modifying treatments clearly impact significantly on MS, and the $\mathrm{ABN}$ recommends starting treatment as early as possible in eligible patients. The complexities and uncertainties that we have outlined, combined with the now significant range of treatments available for relapsing-remitting MS, make treatment decisions complex for both patient and neurologist. We stress the importance of shared conversations about disease activity, risk and benefit, to make the choice that is right for the individual and their circumstances. Furthermore, the ABN believes that people with MS should be managed by neurologists ${ }^{\mathrm{i}}$ with specialist experience of managing patients with this condition and who have the capacity to manage safety monitoring. It is essential that MS specialist neurologists can prescribe the full range of available licensed treatments according to what is clinically appropriate and best meets individual needs.

After considering the evidence, the following summary statements can be made concerning the use of disease-modifying drugs in treating MS:

1. All of the licensed disease-modifying treatments for MS - $\beta$-interferons, glatiramer acetate, fingolimod, teriflunomide, dimethyl fumarate, natalizumab and alemtuzumab-reduce relapse rate and MRI lesion accumulation in relapsing-remitting MS, to varying extents. ${ }^{2}$ There are relatively few comparison trials, ${ }^{3-7}$ and so information on comparative efficacy is usually inferential. Care must be taken with a direct 'inter-trial' comparison of the compounds since the pivotal trials will have occurred at differing time points and there are confounders (known and unknown) - control groups, for example, may differ remarkably between different trials. Nonetheless, we suggest that these seven agents can be divided into two broad classes: drugs of moderate efficacy (average relapse reduction in $30-50 \%$ range), including the $\beta$-interferons, glatiramer, teriflunomide, dimethyl fumarate and fingolimod; and drugs

\footnotetext{
${ }^{\text {i } W e ~ a r e ~ n o t ~ a w a r e ~ o f ~ a n y ~ g e n e r a l l y ~ a c c e p t e d ~ d e f i n i t i o n ~ o f ~ ' m u l t i p l e ~}$ sclerosis specialist neurologist'. Our view is that an effective and safe multiple sclerosis team, whether based at a Regional Neuroscience Centre or a District General Hospital, should include more than one MS specialist consultant neurologist working with an appropriately experienced multidisciplinary health care team. It is very difficult for a single consultant neurologist interested in MS working in isolation to maintain his or her specialist skills in MS.
}

of high efficacy (average relapse reduction substantially more than 50\%), alemtuzumab and natalizumab. Side effect profiles vary considerably between the drugs.

2. Mitoxantrone has significant adverse effects and is not obviously superior in efficacy to the newer drugs of high efficacy. It is not licensed for MS in the UK, but is still used, if infrequently, on an off-label basis.

3. Although it seems plausible that reducing relapse rate and MRI lesion accumulation would favourably influence the long-term prognosis, natural history data show only a weak correlation between long-term disability and relapse frequency. ${ }^{8} 9$ Some trials have reported reduced accumulation of disability over 2-3 years, even with relatively modestly effective drugs such as $\beta$-interferon; ${ }^{10}{ }^{11}$ others have not. ${ }^{12-14}$ Similarly, some uncontrolled long-term data (up to 16 years) suggest the disease-modifying therapies reduce the accumulation of disability; ${ }^{15}$ other studies suggest not. ${ }^{16}{ }^{17}$ There are no published and peer-reviewed controlled trial results showing long-term benefit (although it is very challenging to perform controlled trials over periods longer than 2-3 years).

4. There is a consensus that none of the currently available disease-modifying therapies significantly modifies progressively increasing disability that is unrelated to relapses (progressive non-relapsing MS). ${ }^{18-20}$ It is also not established securely that giving long-term diseasemodifying therapy (a) reduces the accumulation of disability by whatever mechanism or (b) prevents or slows entry to the secondary progressive stage of the disease. The ABN calls for more research addressing these important issues. The implication, however, is that no disease-modifying treatment is effective, or indicated, in patients with established progressive MS in the absence of relapses.

5. It is not yet clear whether treatment should aim for a target such as 'no evidence of disease activity'-either clinical or radiological. At present, there is no evidence upon which to offer guidance-whether patients on treatment experiencing, say, one relapse every five years fare worse over a 10-year time frame than those who have no relapses is not known. Therefore, whether a single relapse should trigger an immediate treatment escalation is not known. Again, more research is needed.

6. New MRI lesions are a more sensitive index of inflammatory disease activity than clinical relapses, occurring up to 10 times more frequently than clinically eloquent relapses. ${ }^{21}$ Accordingly, many now substitute MRI activity for clinical activity in the classification, diagnosis and management of MS. For instance, people previously considered to have a 'clinically isolated syndrome' may now be diagnosed as having MS, under the 2010 McDonald criteria, if there is evidence of spatial and temporal dispersion of MRI lesions. ${ }^{22}$ Recently, the European Medicines Agency has explicitly recognised that MS may be defined as 'active' on radiological or clinical evidence. 
7. There has been significant progress in assessing MRI activity over 12-month intervals, combined with clinical relapse activity, to prognosticate-for example, the modified Rio criteria in patients receiving $\beta$-interferon ${ }^{23}$-so that, ultimately, treatment decisions might be tailored accordingly; the ABN encourages further such research. Meanwhile, particularly in the first years after diagnosis, it is appropriate to consider including MRI scanning during the annual review recommended by the 2014 NICE Clinical Guideline for the management of MS [NICE CG186], both on grounds of monitoring efficacy and (with many agents) safety. By implication, this annual review will need to be conducted by the MS specialist neurologist who is also best placed to determine whether MRI scanning is required.

8. There are now many people with relatively longstanding relapsing-remitting MS who have used $\beta$-interferons or glatiramer for perhaps several years and whose disease is stable clinically. It is unclear whether such patients would benefit from MRI monitoring.

9. Immunotherapies appear particularly helpful when given early to people with active relapsing-remitting disease, before there is fixed disability or secondary progression. For instance, there was dramatic benefit in a clinical trial of a cohort with fewer than 2 years' disease duration, a mean Expanded Disability Status Scale (EDSS) of 2.0, and more than two relapses/year. ${ }^{24}$

10. MS specialist neurologists may adopt either an 'escalation' strategy or an 'induction' strategy in treating MS. The 'escalation' strategy involves starting with the drug that is considered the least toxic but which will control the patient's disease, and escalating to more potent therapies in the face of continued disease activity. An 'induction' strategy involves giving a powerful drug, with significant side effects, early in the disease; an example of this is the alemtuzumab trials. We clearly require long-term data comparing the benefits and risks of these approaches.

11. As newer treatments emerge and when there is clinical equipoise agreed between clinician and patient, and there are clinical trials available for recruitment, then patients should be offered participation in relevant studies.

12. UK health systems have diverged with the devolved administrations, not least in respect of treatment guidelines and/or restrictions. These differences render it now impossible to make treatment recommendations that are simultaneously compliant with all of the relevant advisory or statutory medicines agencies (eg, NICE, Scottish Medicines Consortium). Therefore, this current document takes a pragmatic approach based on best clinical practice and the licensed indications of each therapeutic agent. The ABN continues to support a fundamental principle of the NHS that, despite these changes, all patients have a right to access the appropriate expertise and therapy.

\section{GENERAL MANAGEMENT ADVICE}

Disease-modifying treatment should be started and supervised by an MS specialist neurologist. When considering potential disease-modifying treatment options, it is important that patients and neurologists fully appreciate the risk and benefit of drugs, and of leaving the disease untreated. It is important from the outset to give patients accurate information on the expectations of treatment, including the evidence that disease-modifying treatment efficacy can be only partial, moderate and not curative. Patients should also discuss the risk as well as expected benefit of treatment; monitoring requirements; and work, family and other factors that are personally important, and clinicians should take account of their views in making the treatment selection.

MS specialist nurses play a vital role in ensuring that the treatment pathways are followed, managing symptoms, and providing education, information and reassurance to patients during and between clinic attendances. In many centres, MS specialist nurses play a key role in supporting patients through the process of making choices about treatment options as well as monitoring patients on these often complex treatments.

Patients can also obtain information from patient groups, particularly the Multiple Sclerosis Trust and the Multiple Sclerosis Society, which have produced information leaflets in plain language, as well as a range of leaflets on other symptomatic, psychological and social aspects of living with MS. There are also several excellent websites particularly targeted towards patients, providing valuable information and guidance.

Once started on therapy, patients should remain under the supervision of specialist MS neurologists and nurses; an important aim of this is to encourage adherence to a monitoring protocol tailored to the safety profile and recommended risk monitoring programme of the individualised therapy. Continued supervision by the specialist MS team is essential to assess treatment effectiveness and to document relapses. Different disease-modifying treatments require varied pretreatment investigations and ongoing monitoring, some of which may be devolved to the general practitioner. However, it is important that the MS neurology centre continues to supervise the care of patients on disease-modifying treatments, to identify side effects and assess therapeutic efficacy.

In Europe and the USA, it is common practice to use MRI to monitor disease activity in patients on disease-modifying treatments. This is increasingly part of regular practice in the UK and may help in decisions concerning either the escalation or the stopping of treatments. There is limited direct evidence upon which to base the frequency of imaging, and we require more research on this topic. Serial MRI is particularly important during treatment with natalizumab. Neuroscience centres with expertise in MS will increasingly need ready access to MRI and other investigatory services, such as cardiac monitoring or 
optical coherence tomography, in order to monitor disease-modifying treatments safely.

\section{RECOMMENDATIONS FOR STARTING DISEASE-MODIFYING TREATMENT}

Eligible patients will normally be ambulant (maximum EDSS 6.5). There are no treatments licensed for use during pregnancy (but see below).

As mentioned above, the currently licensed diseasemodifying treatments divide broadly into two classes:

- drugs of moderate efficacy ('Category 1')

- $\beta$-interferons (including 'pegylated' $\beta$-interferon)

- glatiramer acetate

- teriflunomide

- dimethyl fumarate

- fingolimod

- drugs of high efficacy ('Category 2')

- alemtuzumab

- natalizumab

\section{Relapsing-remitting MS}

Patients with relapsing-remitting MS who have had two or more clinical relapses in the previous two years are considered to have 'active' disease that warrants consideration of disease-modifying treatments. Increasingly, clinicians are starting treatments in people whose disease is judged 'active' because of a single recent relapse and/or on radiological grounds, including both patients newly diagnosed according to the 2010 'MacDonald criteria', ${ }^{21}$ and those with longer established disease who develop new MRI lesions without clinical relapse. As mentioned above, the European Medicines Agency has explicitly recognised that disease activity may be established on radiological or clinical grounds (in its licensing of alemtuzumab).

All individuals with active relapsing-remitting MS should be considered expeditiously for treatment. Most are likely to start treatment with a Category 1 drug. It seems likely that dimethyl fumarate and fingolimod are the more effective drugs in this category, with the advantage of being oral agents. Some people with active disease will prefer, together with their neurologist, to start dimethyl fumarate or, if 'highly active', fingolimod in this group. (In some, one of the Category 2 drugs may be appropriate; see below.) $\beta$-Interferon, teriflunomide and glatiramer acetate, which appear broadly to be equally effective, are probably a little less effective than dimethyl fumarate or fingolimod. However, the $\beta$-interferons and glatiramer acetate have been used extensively for decades in MS, and there is a wealth of clinical experience confirming their general safety. Individuals with relatively quiescent disease and/or who are more risk-averse might therefore be more likely to choose one of the $\beta$-interferons or glatiramer acetate. Individuals with needle phobia may choose teriflunomide, dimethyl fumarate or, if eligible, fingolimod. There is increasing information on the long-term safety profile of these newer agents, and their use requires patients to be followed by MS specialist neurologists and nurses; those on 'older' agents also need specialist follow-up to monitor and assess disease activity.

\section{More active relapsing-remitting MS}

Patients may be classified as having more active MS by frequent clinical relapses and/or MRI activity either when untreated or while on a Category 1 drug. The formal criteria for high-disease activity despite interferon- $\beta$ or glatiramer requires one relapse in the previous year on interferon- $\beta$ and either (a) $\geq 1$ gadolinium-enhancing MRI lesions or (b) at least nine T2-hyperintensive lesions on cranial MRI. ${ }^{i i}$ We recommend that patients with more active disease use one of the Category 2 drugs, natalizumab or alemtuzumab. Indirect comparison suggests that alemtuzumab and natalizumab have similar efficacy. Although alemtuzumab's licensed indication is much less restrictive, allowing for anyone to be treated with 'active' MS, defined clinically or radiologically (so allowing alemtuzumab to be used in contexts where natalizumab is not licensed), we recommend that, given its potential adverse effects, alemtuzumab should be mainly confined to patients with more active disease. Alemtuzumab and natalizumab are appropriate where individuals and their MS specialist neurologists are most concerned to achieve high efficacy, despite the more complex safety profile compared to Category 1 drugs.

Some people who have experienced relapses despite using a Category 1 agent may be particularly risk-averse. Others may have had infrequent or occasional minor relapses. In such instances, it may be appropriate to change from one to another Category 1 agent, rather than escalating to a Category 2 drug. In such instances, clinicians should bear in mind the likely greater potency of fingolimod and dimethyl fumarate compared with the interferons, glatiramer and teriflunomide.

Switching between Category 1 agents because of continued disease activity may be justified on the basis of MRI-proven disease activity alone (ie, without clinical relapses). By contrast, switching from a Category 1 agent to a Category 2 monoclonal antibody is probably justifiable only when there is clinical evidence of high-disease activity despite treatment. We recognise, however, that rapid new MRI lesion acquisition, in the absence of clinical relapses, rarely may indicate sufficient disease activity to consider escalation to monoclonal antibodies rarely, despite there being no

\footnotetext{
${ }^{i i}$ In the licensing of natalizumab, 'rapidly evolving severe multiple sclerosis' was defined as $\geq 2$ disabling relapses in previous year (on or off therapy) and $\geq 1$ gadolinium-enhancing MRI lesion or significant increase in MRI T2 lesion load.
} 
clear evidence base for this. We encourage more research into this question.

\section{Clinically isolated syndrome}

Various disease-modifying treatments can delay the diagnosis of MS in patients with a clinically isolated syndrome, ${ }^{25-29}$ though there is less secure evidence for their evidence of long-term benefit. ${ }^{30} 31$ Neurologists may consider advising treatment, after discussion with the patient concerning the risks and benefits, for individuals within 12 months of a significant clinically isolated syndrome, if MRI evidence establishes a diagnosis of MS (2010 McDonald criteria [22]) or predicts a high likelihood of recurrent episodes (ie, development of MS), and perhaps particularly if cerebrospinal fluid examination shows central nervous system-restricted oligoclonal immunoglobulin bands. Currently, only the $\beta$-interferons and glatiramer are licensed for clinically isolated syndrome.

\section{People aged under 18 years}

We believe that minors aged between 16 and 18 years should be treated according to the above guidelines. Children with MS aged $<16$ should be treated in specialist clinics, preferably under a combined team including adult and paediatric neurologists with a particular interest in MS.

\section{Primary or secondary progressive MS}

None of the current disease-modifying treatments is recommended in non-relapsing secondary progressive MS or in primary progressive MS. Some people with relapsing secondary progressive MS, whose relapses are their main cause of increasing disability, may benefit from disease-modifying treatment.

\section{Recommendations for stopping disease-modifying treatment}

Decisions to start or stop treatment, or to perform MRI for diagnosis and management, should recognise the central importance of patient choice; patients should be fully informed of relevant facts and uncertainties before making a decision with their MS specialist neurologist. It can be impossible in individual patients to be certain that a treatment is not helping. We believe it is not feasible to have mandatory stopping criteria that apply in all cases. The difficulty of stopping treatment in people with progressive disease is compounded by the absence of alternative options for disease modification-but this is no argument for continuing an ineffective (and expensive) treatment. Clinicians should consider stopping disease-modifying treatment in the following scenarios:

1. Significant side effects specific to any individual agent should trigger withdrawal of that agent and consideration of an alternative treatment.

2. Development of non-relapsing secondary progressive MS.
3. Pregnancy. During pregnancy, disease-modifying treatments should normally be stopped, as stated in the summary of product characteristics. In anticipation of a planned pregnancy, patients should be advised that there is limited information on the risks of early fetal exposure to some disease-modifying treatments, while others have known teratogenicity. We therefore recommend that women stop disease-modifying treatments while trying to conceive unless, in the neurologist's opinion, the woman's clinical condition requires treatment. Given the increased risk of relapse in the puerperium, treatment should be restarted early after delivery, depending on discussions concerning breast feeding.

\section{Author affiliations}

${ }^{1}$ Bristol Institute of Clinical Neurosciences, Learning and Research Building, Southmead Hospital, Bristol, UK ${ }^{2}$ Department of Neurology, St George's University Hospital, London, UK

${ }^{3}$ Department of Neurology, Basingstoke Hospital, Basingstoke, UK

${ }^{4}$ Department of Neurology, National Hospital for Neurology and Neurosurgery, University College,

London, UK

${ }^{5}$ Department of Neurology, Queen's Hospital, Romford, UK

${ }^{6}$ Department of Neurology, University of Cambridge, Cambridge, UK.

${ }^{7}$ Department of Neurology, Barts and The London

School of Medicine and Dentistry, London, UK

${ }^{8}$ Department of Neurology, National Hospital for

Neurology and Neurosurgery, University College,

London, UK

${ }^{9}$ Department of Neurology, Hurstwood Park

Neurological Centre, Haywards Heath

${ }^{10}$ Department of Neurology, Department of Neurology,

Barts and The London School of Medicine and

Dentistry, London, UK

${ }^{11}$ Department of Neurology, University Hospital,

Coventry, UK

${ }^{12}$ Department of Neurology, Kings' College Hospital,

London, UK

${ }^{13}$ Department of Neurology, The Walton Hospital, Liverpool, UK

${ }^{14}$ Department of Neurology, University of Plymouth, Plymouth, UK

Acknowledgements We thank the MS Society and MS Trust whose very helpful contributions during consultation on the prescribing guideline aided its development.

Competing interests The Association of British Neurologists has received sponsorship from Bayer, Biogen Idec, Genzyme, Merck Serono, Novartis, Pfizer, and Teva within the past five years; and conference exhibition income from Actelion, Allergan, Baxter Healthcare, Beacon Pharmaceuticals, Biomarin,

Boehringer Ingelheim, Eli Lilly, Eisai, EyeBrain, Genus Pharma, Glaxosmith Kline, Grifols UK Ltd, Ipsen, Jansen, Meda

Pharma, Medtronic, Pfizer, UCB, Walters Cluwer and

Wisepress. NS has received financial support for research and for educational purposes, and hospitality, from

Genzyme-Sanofi, Merck-Serono, Biogen, Teva, Bayer and 
Novartis; and through NJS North Bristol NHS Trust and/or the University of Bristol has also received financial support for capital projects from these companies. SC has received honoraria and served on advisory boards for Biogen and Bayer, and received travel support from Novartis and Biogen. JC receives support from the National Institute of Health Research (NIHR), University College London Hospitals (UCLH)/UCL Biomedical Research Centre (BRC) funding scheme. He has taken part in conferences/consultancy sponsored by Novartis, Teva and EMD Serono. ACh has received travel grants or sponsorship for attending medical congresses, speaker fees and honoraria from: Novartis, Biogen Idec, Bayer-Schering, UCB, Eisai, Terumo BCT and Genzyme; he has also received payments from the medical market research groups for ad hoc consultancy. ACo has received travel expenses and honoraria, and his institution has received research support and donations, from Genzyme (a Sanof company). GG has received travel expenses, hospitality, consultancy fees or honoraria from AbbVie, Biogen-Idec, GSK, Merck-Serono, Novartis, Teva, Genzyme-Sanofi, Roche and/or Synthon. DM has received (through payments to UCL Institute of Neurology) honoraria for Advisory Committee and/or Consultancy advice from Biogen Idec, GlaxoSmithKline, Novartis, Merck, Chugai, Mitsubishi Pharma Europe and Bayer Schering Pharma, and compensation for performing MRI analysis from

GlaxoSmithKline, Biogen Idec, Novartis and Merck. The Queen Square MS Centre at UCL Institute of Neurology is supported by the UK MS Society and UCL-UCLH Biomedical Research Centre. WR has received financial support for research and educational purposes, hospitality, fees for speaking and advisory boards, from Biogen Idec, Genzyme, Novartis, Roche, Merck Sereno and Bayer. KS is a PI on trials sponsored by Novartis, Roche and Teva. He has received speaking honoraria from, and/or served on advisory boards for, Novartis, Biogen, Teva, Merck-Serono and Merck Inc, and has received travel support from Genzyme. Through KS, Queen Mary, University of London has received funding for research from Novartis, and scientific meeting support from Biogen, Teva and Genzyme. AS has received sponsorship or honoraria from Biogen Idec, Bayer, Genzyme, Serono, Teva and Novartis. ES has acted as an advisor or received financial support for research and for educational purposes, and hospitality, from Merck-Serono, Biogen, Teva, Bayer-Schering and Novartis; and through his NHS trust has also received financial support for projects/ service developments from some of these companies. $\mathrm{He}$ has been an investigator in commercial trials sponsored by Biogen Idec, Novartis, Teva, Receptos, Roche, GW Pharma and GSK. CY is a PI on trials sponsored by Biogen, Novartis, Roche, Sanofi and Teva, and has received financial support for research and educational purposes, hospitality, fees for speaking and advisory boards, from Biogen Idec, Genzyme, Novartis, Roche and Teva.

Provenance and peer review Commissioned; externally peer reviewed.

\section{REFERENCES}

1 HSC 2002/004-Cost effective provision of disease modifying therapies for people with Multiple Sclerosis. Health Do. London. 2002.

2 Rice CM. Disease modification in multiple sclerosis: an update. Pract Neurol 2014;14:6-13.

3 Vermersch P, Czlonkowska A, Grimaldi LM, et al. Teriflunomide versus subcutaneous interferon beta- $1 \mathrm{a}$ in patients with relapsing multiple sclerosis: a randomised, controlled phase 3 trial. Mult Scler 2014;20:705-16.

4 Mikol DD, Barkhof F, Chang P, et al. Comparison of subcutaneous interferon beta- $1 \mathrm{a}$ with glatiramer acetate in patients with relapsing multiple sclerosis (the REbif vs Glatiramer Acetate in Relapsing MS Disease [REGARD] study): a multicentre, randomised, parallel, open-label trial. Lancet Neurol 2008;7:903-14.
5 Fox RJ, Miller DH, Phillips JT, et al. Placebo-controlled phase 3 study of oral BG-12 or glatiramer in multiple sclerosis. N Engl J Med 2012;367:1087-97.

6 Cohen JA, Coles AJ, Arnold DL, et al. Alemtuzumab versus interferon beta $1 \mathrm{a}$ as first-line treatment for patients with relapsing-remitting multiple sclerosis: a randomised controlled phase 3 trial. Lancet 2012;380:1819-28.

7 Cohen JA, Barkhof F, Comi G, et al. Oral fingolimod or intramuscular interferon for relapsing multiple sclerosis. N Engl J Med 2010;362:402-15.

8 Scalfari A, Neuhaus A, Daumer M, et al. Early relapses, onset of progression, and late outcome in multiple sclerosis. JAMA Neurol 2013;70:214-22.

9 Confavreux C, Vukusic S, Moreau T, et al. Relapses and progression of disability in multiple sclerosis. $\mathrm{N} \mathrm{Engl} \mathrm{J} \mathrm{Med}$ 2000;343:1430-8.

10 Jacobs LD, Cookfair DL, Rudick RA, et al. Intramuscular interferon beta-1a for disease progression in relapsing multiple sclerosis. Ann Neurol 1996;39:285-94.

11 Group PS. Randomised double-blind placebo-controlled study of interferon beta-1a in relapsing/remitting multiple sclerosis. Lancet 1998;352:1498-504.

12 The IMSSG. Interferon beta- $1 \mathrm{~b}$ is effective in relapsing-remitting multiple- sclerosis-clinical-results of a multicenter, randomized, double- blind, placebo-controlled trial. Neurology 1993;43:655-61.

13 Panitch H Miller A, Paty D, et al. Interferon beta-1b in secondary progressive MS: results from a 3-year controlled study. Neurology 2004;63:1788-95.

14 Secondary Progressive Efficacy Clinical Trial of Recombinant Interferon-Beta-1a in MSSG. Randomized controlled trial of interferon- beta-1a in secondary progressive MS: Clinical results. Neurology 2001;56:1496-504.

15 Bermel RA, Weinstock-Guttman B, Bourdette D, et al. Intramuscular interferon beta-1a therapy in patients with relapsing-remitting multiple sclerosis: a 15-year follow-up study. Mult Scler 2010;16:588-96.

16 Ebers GC, Traboulsee A, Langdon D, et al. Analysis of clinical outcomes according to original treatment groups 16 years after the pivotal IFNB-1b trial after the pivotal IFNB-1b trial. J Neurol Neurosurg Psychiatry 2010;81:907-12.

17 Greenberg BM, Balcer L, Calabresi PA, et al. Interferon beta use and disability prevention in relapsing-remitting multiple sclerosis. JAMA Neurol 2013;70:248-51.

18 Leary SM, Miller DH, Stevenson VL, et al. Interferon beta-1a in primary progressive MS: an exploratory, randomized, controlled trial. Neurology 2003;60:44-51.

19 Montalban X, Sastre-Garriga J, Tintore M, et al. A single-center, randomized, double-blind, placebo-controlled study of interferon beta-1b on primary progressive and transitional multiple sclerosis. Mult Scler 2009;15:1195-205.

20 Wolinsky JS, Narayana PA, O'Connor P, et al. Glatiramer acetate in primary progressive multiple sclerosis: results of a multinational, multicenter, double-blind, placebo-controlled trial. Ann Neurol 2007;61:14-24.

21 Kappos L, Moeri D, Radue EW, et al. Predictive value of gadolinium-enhanced magnetic resonance imaging for relapse rate and changes in disability or impairment in multiple sclerosis: a meta-analysis. Gadolinium MRI Meta-analysis Group. Lancet 1999;353:964-9.

22 Polman CH, Reingold SC, Banwell B, et al. Diagnostic criteria for multiple sclerosis: 2010 revisions to the McDonald criteria. Ann Neurol 2011;69:292-302. 


\section{ABN GUIDELINES}

23 Sormani MP, Rio J, Tintore M, et al. Scoring treatment response in patients with relapsing multiple sclerosis. Mult Scler 2013;19:605-12.

24 Coles AJ, Compston DA, Selmaj KW, et al. Alemtuzumab vs. interferon beta-1a in early multiple sclerosis. N Engl J Med 2008;359:1786-801.

25 Kappos L, Polman CH, Freedman MS, et al. Treatment with interferon beta- $1 \mathrm{~b}$ delays conversion to clinically definite and McDonald MS in patients with clinically isolated syndromes. Neurology 2006;67:1242-9.

26 Comi G, Filippi M, Barkhof F, et al. Effect of early interferon treatment on conversion to definite multiple sclerosis: a randomised study. Lancet 2001;357:1576-82.

27 Jacobs LD, Beck RW, Simon JH, et al. Intramuscular interferon beta-1a therapy initiated during a first demyelinating event in multiple sclerosis. CHAMPS Study Group. N Engl J Med 2000;343:898-904.

28 Comi G, Martinelli V, Rodegher M, et al. Effect of glatiramer acetate on conversion to clinically definite multiple sclerosis in patients with clinically isolated syndrome (PreCISe study): a randomised, double-blind, placebo-controlled trial. Lancet 2009;374:1503-11.

29 Miller AE, Wolinsky JS, Kappos L, et al. Oral teriflunomide for patients with a first clinical episode suggestive of multiple sclerosis (TOPIC): a randomised, double-blind, placebo-controlled, phase 3 trial. Lancet Neurol 2014;13:977-86.

30 Kinkel RP, Dontchev M, Kollman C, et al. Association between immediate initiation of intramuscular interferon beta- $1 \mathrm{a}$ at the time of a clinically isolated syndrome and long-term outcomes: a 10-year follow-up of the Controlled High-Risk Avonex Multiple Sclerosis Prevention Study in Ongoing Neurological Surveillance. Arch Neurol 2012;69:183-90.

31 Kappos L, Freedman MS, Polman CH, et al. Long-term effect of early treatment with interferon beta- $1 \mathrm{~b}$ after a first clinical event suggestive of multiple sclerosis: 5 -year active treatment extension of the phase 3 BENEFIT trial. Lancet Neurol 2009;8:987-97. 